\title{
Correction to: Mass spectrometry-based proteomic capture of proteins bound to the MACC1 promoter in colon cancer
}

\author{
Yahui Huang ${ }^{1,2,4} \cdot$ Yi Xiang $^{2}$ - Zhongpeng Xie ${ }^{1,2,5} \cdot$ Yuxiang Cai ${ }^{1,2}$. Qiongzhi Yang ${ }^{1,2} \cdot$ Huichao Huang $^{3}$. \\ Zhuchu Chen ${ }^{3} \cdot$ Zhefeng Xiao $^{3}$. Qiongqiong He ${ }^{1,2}$
}

Published online: 4 September 2020

(c) Springer Nature B.V. 2020

\section{Correction to: \\ Clinical \& Experimental Metastasis (2020) 37:477-487 \\ https://doi.org/10.1007/s10585-020-10045-z}

In the original publication of the article, Acknowledgements section was published incorrectly. The correct Acknowledgements is given in this Correction.

Acknowledgements We thank the State Key Basic Research Development Plan (2011CB910700 microenvironmental proteomics of solid tumors) and the Teacher Research Fund of Central South University. We also thank the Department of Pathology, Xiangya Hospital, Central South University for technical support. We also thank Prof. Hodaka Fujii for providing 3xFLAG-dCas9/pCMV-7.1 as a gift and thank

Yahui Huang and Yi Xiang have contributed equally to this work and should be considered co-first authors.

The original article can be found online at https://doi.org/10.1007/ s10585-020-10045-z.

Zhefeng Xiao

xiaozf@csu.edu.cn

$\triangle$ Qiongqiong $\mathrm{He}$

qiongqionghe@csu.edu.cn

1 Department of Pathology, Xiangya Hospital, Central South University, Changsha, Hunan, People's Republic of China

2 School of Basic Medical Sciences, Central South University, Changsha, Hunan, People's Republic of China

3 NHC Key Laboratory of Cancer Proteomics, Xiangya Hospital, Central South University, Changsha, Hunan, People's Republic of China

4 Department of Pathology, Xuchang Central Hospital, Henan University of Science and Technology, Xuchang, Henan, People's Republic of China

5 Department of Pathology, Hainan General Hospital, Haikou, Hainan, People's Republic of China his lab's patented technology of adopting the CRISPR complexes to isolate specific DNA regions.

Publisher's Note Springer Nature remains neutral with regard to jurisdictional claims in published maps and institutional affiliations. 